\title{
Privacy Concerns in Enterprise Social Travel: Attitudes and Actions
}

\author{
Netta Aizenbud-Reshef, Artem Barger, Yael Dubinsky, \\ Ido Guy, and Shiri Kremer-Davidson \\ IBM Haifa Research Lab \\ Haifa, Israel \\ \{neta, bartem, dubinsky, ido, shiri\}@il.ibm.com
}

\begin{abstract}
Privacy in travel refers to the way people manage their personal travel information and their willingness to share this information with others. Travel privacy concerns affect the amount and type of information people are willing to share within social networks, before or after their trip. Our study focuses on privacy and sharing concerns regarding business travel in an enterprise setting. We briefly present Voyage, an enterprise social travel application, and describe a study based on qualitative and quantitative data that inspects privacy and sharing concerns in business travels. We found that most employees are theoretically willing to share their business travel plans, but in practice they share less than expected. Further, most employees are less concerned to share their past travel information than their future plans. Based on our study, we suggest guidelines for the development of location based and enterprise travel applications.
\end{abstract}

Keywords: Privacy, business travel, information sharing, social activity.

\section{Introduction}

Sharing travel information is becoming very popular on the web. People typically share their opinions and experiences about places they visited, hotels they stayed in, and services they used; this is done on web sites such as TripAdvisor.com, Hotels.com, and yelp.com. Recently, people have also begun sharing their itinerary details with a select list of friends (defined on the site), through services such as TripIt.com and Dopplr.com. The benefits of sharing travel plans are clear as people can learn from other individuals' experiences and make better informed decisions [6, 11]. Ultimately, these decisions can affect their itinerary, cost, and level of satisfaction or enjoyment. Sharing travel plans also facilitates better coordination with other people and increases awareness of those who plan to be at the same place.

Privacy in the context of social applications that support information sharing has already been studied. Ahern et al. [1] examined privacy decisions in mobile and online photo sharing and identified relationships between the location of photo captures and photo privacy settings. Similarly, other studies [2, 3, 8] explored whether and what users are willing to disclose about their location. For instance, it was found 
that participants typically disclosed the most useful details about their location or did not disclose their location at all [2]. General studies of the impact of privacy concerns in social communities concluded that trust within the community and sharing norms affect information sharing [5, 9]. Because an enterprise setting can be readily perceived as a community, similar behavior is expected for business travel as well.

There is often a gap between what people want to get from other's experience and their willingness to contribute [7]. In an enterprise setting, this gap seems to be less pronounced. Inside the enterprise, people are willing to share more information than on a public site, because they are less concerned about the ramifications of sharing personally identifying information [4].

Our motivation is to study privacy concerns in enterprise social travel, the willingness of employees to share and consume travel data, and the main factors affecting their decision to share. As many business processes are turning social these days, we believe that enterprise social travel is one example of an enterprise business process that is transforming to be more social than before. The implications presented in this paper are relevant for such business processes as well.

The paper is organized as follows: first we present Voyage, a social enterprise travel application. Then we describe the research setting, including the methods we used for gathering data. Following this, we analyze the results and conclude with a summary and description of future work.

\section{Voyage - Travel Meets Social}

We experiment with Voyage, an enterprise travel reservation tool that aims at making the travel reservation process more social and collaborative. It extends a common enterprise web-based travel reservation application (such as Expedia.com, Orbitz.com, etc.) and adds various functional and social features, such as:

Learn from past travels - past reservations are aggregated to show statistical measures, such as the percentage of people renting a car in a specific city, the most frequently reserved hotel, or the flight route usually taken when traveling from one place to another.

Share feedback - users can provide feedback (comments and ratings) and see information entered by others for any travel-related item in their itinerary, such as hotels, flights, airlines, car agencies, airports, places, etc.

Share travel plans - users can share their itineraries and select the sharing level for each itinerary by setting its privacy level. Itineraries defined as 'Private' are not exposed to anyone but the user; those defined as 'Public' are visible to everyone. 'Confidant' itineraries are exposed only to a chosen set of users. This enables users to collaborate on travel planning, see who is going to visit the same place, see what reservations were made by others, and so forth. Users can also define a default privacy level to be used for all of their travel plans. The default privacy level set by the system is Public, in order to encourage sharing in the enterprise.

Travel information in one place - travel-related information is aggregated from both publicly available sources (such as weather sites and sites providing electricity 
information) and enterprise-specific sources (such as a meal limit database or a list of allowed hotels) and presented in one place, instead of sending users around the Internet and company intranet to collect it.

The figures below provide some examples of how the above features are presented on the Voyage site. Fig 1 shows the outcome of a hotel search within a specific period of time. It includes one hotel from the returned result list. Along with general hotel details such as name, address, and price, there are several social features: on the right, enclosed in a red rectangle, are three cues showing the number of comments for this hotel (1), the percentage of reservations made to this hotel out of all hotel reservations in the city $(23 \%)$, and the number of people that already reserved this hotel during part or all of the selected time period (0). In addition, the stars on the left show the hotel average rating ( 3 of 5); more detailed ratings and comments are presented when opening the corresponding sections marked as (b).

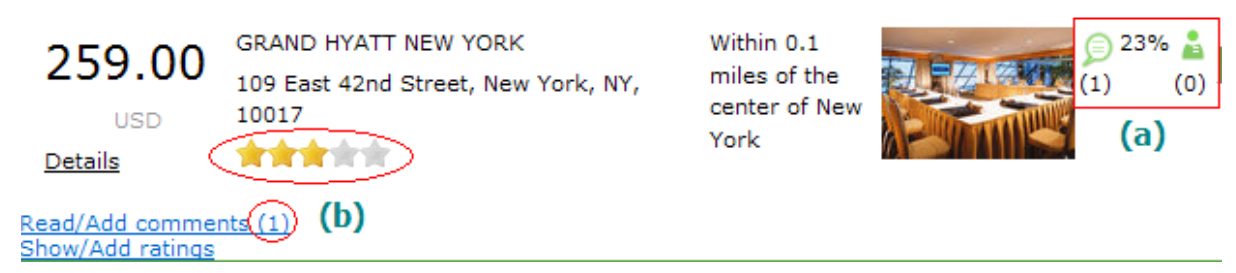

Fig. 1. Hotel options augmented with social cues

Fig 2 shows information on one itinerary (name, dates, and reservation types) and the people with whom it is shared.

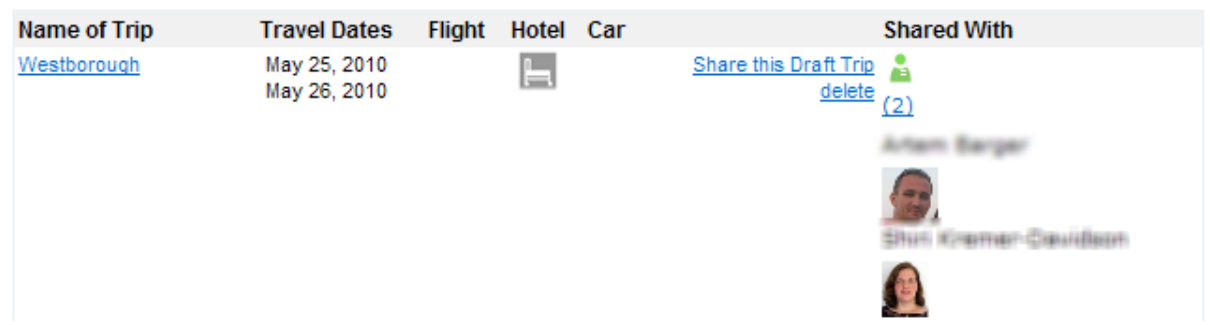

Fig. 2. Sharing travel plans

\section{Travel Sharing and Privacy Concerns}

Our research was conducted within a large distributed organization, with data collected from employees in Asia, Europe, the Middle East, and North America. We used four different methods to gather data about people's travel privacy/sharing concerns in order to increase the findings' trustworthiness: (1) Before Voyage was implemented, an initial user survey was sent to frequent travelers, querying their willingness to share their travel plans and the benefits they envisioned in knowing about others' past reservations and future plans. The survey included 15 multiple- 
choice questions and was answered by 1431 employees; (2) After Voyage was deployed and employees started to use it for their travel planning, we conducted a usage survey asking them about the actions they took while planning. This survey included 16 multiple-choice questions in total, but was adjusted to align with the selections made by the employee in the tool, for example, if the employee defined the privacy level to be 'Private', a question about the concerns that led him to this decision was included in the survey. Out of 92 employees who made their travel planning through Voyage, 54 answered the survey; (3) We inspected the tool logs to determine the actual sharing decisions users made throughout the process. Logs include information on the user actions, specific parameters for each action, the user name, and a time and date stamp; (4) Finally, we conducted semi-structured qualitative interviews with 13 employees, exploring their travel privacy/sharing concerns.

The analysis combines qualitative and quantitative results, which involve comparison of triangulated data sources and thick narrative to strengthen each finding [10]. We divide the results into three aspects. The first aspect deals with concerns and attitudes towards sharing travel information among employees. The second aspect explores the results related to sharing resources, rather than information. We examine sharing controls as the third aspect.

Information Sharing. From the initial survey we learned that employees value information about past travels of other employees: $81 \%$ of employees who answered the initial survey indicated it is useful for them to know where other people from the enterprise usually stay; $69 \%$ think it is useful to know who recently visited their travel destination; and $69 \%$ value ratings and comments input by other employees. From interviews, we learned that before they travel, employees often consult with their colleagues about hotels, directions, leisure activities, and even who they can get together with. One of our interviewees noted: "If it is a place I rarely go or have never been before, I will ask others that have been there or the local people about hotel and means of transportation."

Employees also found value in information on others' future plans, e.g., for coordination: in the initial survey $70 \%$ stated it is beneficial to know what flight a colleague is going to take; $70 \%$ want to know who else is attending a meeting/conference/event, etc. On average, around $70 \%$ of employees find this type of information very useful, while only $2 \%$ indicated it as not useful at all.

The benefits from knowing what other employees do with regard to travel motivate employees to share their travel plans in the majority of cases. Table 1 summarizes data that was gathered in the initial survey and depicts the willingness of employees to share their travel information with anyone in the enterprise (Anyone), their social network (SN), specific people (Specific), or not at all (No one). It is evident from the data in the table that employees are willing to share their travel information with different groups of employees in $96 \%$ of the cases.

In the interviews employees raised different reasons for sharing. Some perceive business travel as belonging to the enterprise, as one of the respondents indicated: "What I do at work belongs to work; I get a salary for that, thus my business travel also belongs to the company". Some see the benefits of coordinating plans: "Maybe someone else is also traveling there in the same week. It's good just to know about it; maybe we won't share anything, like a taxi, but we could go out to eat together." 
Table 1. Sharing travel plans

\begin{tabular}{|c|c|c|c|c|}
\hline \multicolumn{5}{|c|}{ Future plans } \\
\hline Flight & $32 \%$ & $35 \%$ & $28 \%$ & No one \\
\hline Hotel & $34 \%$ & $39 \%$ & $24 \%$ & $4 \%$ \\
\hline City & $29 \%$ & $37 \%$ & $28 \%$ & $6 \%$ \\
\hline \multicolumn{5}{|c|}{ Past travels } \\
\hline Flight & $45 \%$ & $34 \%$ & $18 \%$ & $3 \%$ \\
\hline Hotel & $48 \%$ & $37 \%$ & $13 \%$ & $3 \%$ \\
\hline City & $47 \%$ & $35 \%$ & $14 \%$ & $3 \%$ \\
\hline
\end{tabular}

Another noted "if several of us travel together, it's important that we coordinate: flights and landing times, car rental pooling and selection of car size, hotels to stay in, and so on."

Focusing on the 'Anyone' column of the table, Fig. 3 highlights the difference in willingness to share future plans vs. past travels (on average $47 \%$ vs. 32\%). We confirmed the significance of this difference using One-Way ANOVA $(\mathrm{p}<0.01)$. From interviews we learned that employees are mostly concerned about security issues, for example, that people will know where they are and when they are away from home. As one of the interviewees noted, "The enterprise is very big, complex, and diverse; you can't trust all the people". This concern relates to future plans only and may explain the difference in willingness to share past travels vs. future plans, that is noted before. A somewhat similar concern was expressed in connection with revealing personal information, such as a credit card number: "What if a crook from the enterprise calls the hotel and asks for a copy of the receipt, and my personal information is there (e.g., who I called, my credit card number, etc.)?"

Some interviewees noted that sharing information about their travel also reveals information about other people involved in the trip, such as accompanying people or the customer they are visiting, without checking the concerns these people might have: "Sometimes the confidentiality of the project I am working on restricts the people I can meet."

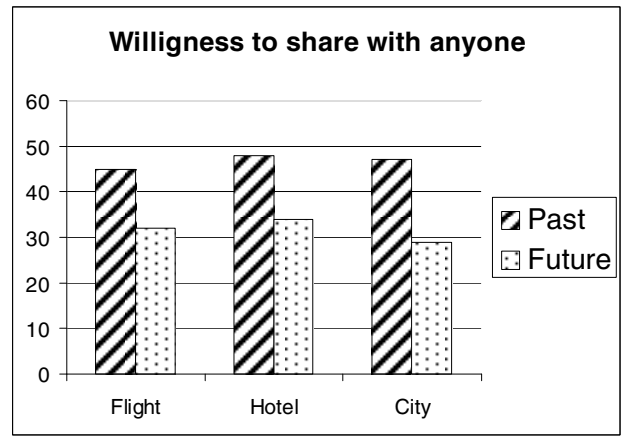

Fig. 3. Past vs. future sharing 


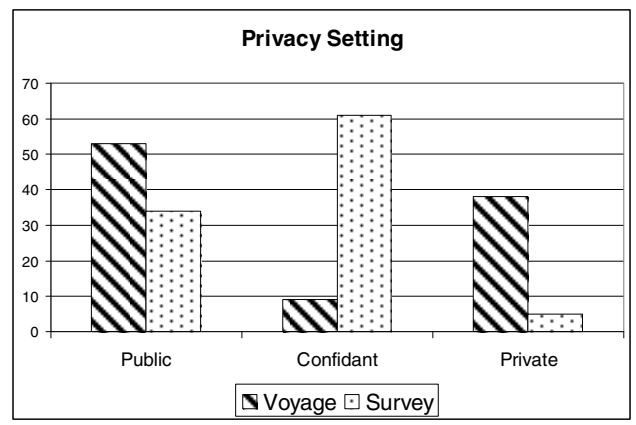

Fig. 4. Privacy setting

Inspecting actual privacy settings of travel reservations made in Voyage, we found that employees were less open to sharing than indicated in the initial survey. These gaps are depicted in Fig. 4. Fewer people than expected defined their trip privacy as 'Confidants'. It might resulted from them not being sure what is the meaning of this privacy level, being presented with the 'Confidants' concept at the first time. It could also result from laziness, since they had to manually define the list of confidants. The big gap between the percentage of people who did not want to share their travel plans according to the survey (5\%) and those that defined their trip to be private $(38 \%)$ is harder to explain. We can only hypothesize that when asked in a survey about attitudes, people tend to be more open than when they are confronted with the actual travel reservation and must make a real decision. We observed a similar phenomenon in one of the interviews. The interviewee first declared that he is willing to share all his travel plans with anyone in the enterprise. Thinking it over and raising some of the above concerns, he withdrew his original statement and changed it to sharing with only specific people.

Nevertheless, employees were willing to expose more about their travel details after they returned from a trip. In the usage survey, $60 \%$ of Voyage users who defined their trip as private, agreed to change it to public after they returned from the trip. This reinforces our findings about the difference between sharing past travels and future plans.

Resource Sharing. Sharing travel resources, such as rented cars or hotels, is treated differently by employees than sharing travel information. When asked about sharing a car rental or a taxi in the initial survey, $35 \%$ of the employees indicated they would share with anyone in the enterprise; $45 \%$ would share only with known people; and the other $20 \%$ are not willing to share at all. We observe that employees are more reluctant to share resources $(20 \%)$ than to share travel plans $(4 \%)$. It is very likely that this results from the feeling that sharing data is less personal than physically being at the same place with a stranger. Employees also feel that it introduces another difficulty to their travel planning: "It's a burden when you have to coordinate a car rental between two people just because they are going to be in the same place". They are willing to share a car if it does not interfere with their plans: "My experience of sharing a taxi has not always been positive, because sometimes you have to wait longer than if you had been alone." 
Sharing Controls. In the initial survey, we queried employees about the importance of controlling over what they are sharing. Table 2 presents the distribution of answers regarding both the control over sharing defaults and the ability to adjust sharing preferences on an item-by-item basis.

Table 2. Sharing controls

\begin{tabular}{|l|c|c|c|c|}
\hline & Critical & Important & $\begin{array}{c}\text { Slightly } \\
\text { important }\end{array}$ & $\begin{array}{c}\text { Not } \\
\text { important }\end{array}$ \\
\hline Control defaults & $16 \%$ & $45 \%$ & $31 \%$ & $8 \%$ \\
\hline Control item-by-item & $15 \%$ & $46 \%$ & $32 \%$ & $7 \%$ \\
\hline
\end{tabular}

As is evident from the table, employees are keen to control the travel information they share. Currently, Voyage supports privacy control over a full itinerary and not for each itinerary item separately. This might explain why more employees than expected chose to define their travel plans as private.

The desire of employees to control the sharing defaults in the system is also clear from the table. Inspecting the behavior of Voyage users, only $30 \%$ opted to set their default privacy level. Out of those that cared do so, 2.5\% set it to 'Private', $5 \%$ set it to 'Confidants', and the rest (92.5), although opted to set their default privacy level, selected to leave it 'Public' as it was defined by default. These findings strengthen previous research works that show that users rely on the system defaults. In our case, the default setting plays even a smaller role, as users can set the privacy level for each trip separately.

\section{Summary}

In this paper, we study travel privacy concerns and the willingness of employees to share their travel plans with others in an enterprise setting. We found that employees value other people's travel information. Although they theoretically agree to share their own travel information, in practice they share less than stated. Employees raised various sharing concerns related to privacy, security, and project confidentiality. We found that there is a difference between sharing past travels and future plans employees are willing to share more of their past travels. To the best of our knowledge, this is the first study to point out this distinction. Future research should examine whether it applies to other business processes as well.

Based on the finding of this study, we suggest two high-level development guidelines for enterprise travel applications. First, employees stated that they would like to have the option to control the level of sharing allowed by the reservation application, and to some level used the options provided by Voyage. As only parts of the itinerary might be confidential, users should be provided with privacy settings to allow them to finely control what information is shared and with whom. Second, employees expressed their will to relax the privacy level of their trip after some time has passed, as the sensitivity of exposing to a larger audience fades over time. We believe that this approach applies not only to travel plans, but for any type of media in general. To encourage sharing, we suggest the application would actively offer users 
to relax the privacy settings of their media after some time has gone by since posting it (for example, issue an alert after a week).

In future work, we intend to further explore how sharing attitudes and actions change over time or in different situations, and the differences in privacy concerns and behavior between business travel and personal travel. We also want to research privacy aspects in other types of business processes and investigate whether the implications, as presented in this paper, are similar.

\section{References}

1. Ahern, S., Eckles, D., Good, D., King, S., Naaman, M., Nair, R.: Over-exposed?: Privacy Patterns and Considerations in Online and Mobile Photo Sharing. In: Proc. ACM Conference on Human Factors in Computing Systems, CHI (2007)

2. Consolvo, S., Smith, I.E., Matthews, T., Lamarca, A., Tabert, J., Powledge, P.: Location Disclosure to Social Relations: Why, When, \& What People Want to Share. Proc. ACM Conference on Human Factors in Computing Systems, CHI (2005)

3. Church, K., Neumann, J., Cherubini, M., Oliver, N.: SocialSearchBrowser: A Novel Mobile Search and Information Discovery tool. In: Proc. IUI, pp. 101-110 (2010)

4. DiMicco, J., Millen, D.R., Geyer, W., Dugan, C., Brownholtz, B.: Caring, Climbing, and Campaigning: Motivations for Social Networking at Work. In: Proc. CSCW (2008)

5. Dwyer, C., Hiltz, S., Passerini, K.: Trust and Privacy Concern within Social Networking Sites: A Comparison of Facebook and MySpace. In: Proc. AMCIS (2007)

6. Gretzel, U., Yoo, K.H.: Use and Impact of Online Travel Reviews. In: Proc. International Conference on Information and Communication Technologies in Tourism, pp. 35-46 (2008)

7. Gretzel, U., Yoo, K.H.: Influence of personality on travel-related consumer generated media creation. Computers in Human Behavior (2010)

8. Gross, R., Acquisti, A.: Information Revelation and Privacy in Online Social Networks. In: Workshop on Privacy in the Electronic Society. ACM Press, New York (2005)

9. Nov, O., Wattal, S.: Social Computing Privacy Concerns: Antecedents \& Effects. In: Proc. ACM Conference on Human Factors in Computing Systems, CHI, pp. 333-336 (2009)

10. Patton, M.Q.: Qualitative Research \& Evaluation Methods, 3rd edn. Sage, Thousand Oaks (2002)

11. Surowiecki, J.: The Wisdom of Crowds. Anchor (2005) 